\title{
Thermodynamics Of dilaton-axion black holes
}

\author{
Tanwi Ghosh* and Soumitra SenGupta ${ }^{\dagger}$ \\ Department of Theoretical Physics , \\ Indian Association for the Cultivation of Science, \\ Jadavpur, Calcutta - 700 032, India
}

\begin{abstract}
Considering a generalised action for Einstein Maxwell theory in four dimensions coupled to scalar and pseudo-scalar fields, the thermodynamic properties of asymptotically flat black holes solutions in such a background are investigated. Bekenstein-Hawking area-entropy law is verified for these class of black holes. From the property of specific heat, it is shown that such black holes can be stable for certain choice of the parameters like charge, mass and the scalar vacuum expectation value. The possibility of a black hole phase transition is discussed in this context.
\end{abstract}

\section{Introduction}

Theories of gravitation with background scalar and pseudo-scalar field have been studied extensively for a long time. In particular in string inspired models, the electromagnetic-dilaton coupled black hole solutions obtained by Garfinkle,Horowitz, Strominger initiated intense activities in these areas 1]. Among various properties of such black hole solutions, the study of their thermodynamic properties has always been an important area of research in the Physics of black holes.

String theory based-models contain two massless scalar fields, dilaton and axion, in the low energy effective action in four dimension. The axion $\zeta$ is connected via a duality transformation to the three form field strength $H_{\mu \nu \lambda}$ corresponding to the two form Kalb-Ramond antisymmtric tensor field $B_{\mu \nu}$ which appears as a massless closed string mode. While in the low energy action the dilaton and axion couple to the electromagnetic field in a specific way, a more generalised coupling with Einstein and Maxwell theory in four dimensions were proposed both for asymptotically flat and non-flat dilaton-axion black holes. The couplings in general depend on the vacuum expectation values of the various moduli of compactification. Using the duality symmetry in string theory it has been shown that starting from one particular spacetime solution with dilaton and axion one can generate inequivalent solutions for the spacetime as well as dilaton and axion. Corresponding black hole solutions have also been studied extensively [2, 3, 4, 4, 5, 6, 7]. This prompted a parametric generalization of this coupling and treat them as independent parameters to study various generic scalar coupled black hole solutions [8]. It is worthwhile therefore to explore various thermodynamic properties of this class of black holes and verify the Bekenstein-Hawking area-entropy conjecture in this context. Moreover as the parameters $a$ and $b$ give us the relative strength of the scalar and pseudo scalar couplings, we propose to investigate in this work, how these couplings modify the thermodynamic properties of these black holes.

The generalized Einstein-Maxwell -dilaton-axion action is given as [8];

$$
S=\int d^{4} x \sqrt{-g}\left[\frac{1}{2 \kappa}\left(R-\frac{1}{2} \partial_{\mu} \varphi \partial^{\mu} \varphi-\frac{1}{2} e^{2 a \varphi} \partial_{\mu} \zeta \partial^{\mu} \zeta\right)-e^{-a \varphi} F_{\mu \nu} F^{\mu \nu}-b \zeta F_{\mu \nu} * F^{\mu \nu}\right]
$$

where $a$ and $b$ are coupling parameters of dilaton field $\varphi$ and axion field $\zeta$ respectively with the Maxwell field tensor $F_{\mu \nu}$ and its dual $* F^{\mu \nu}$. The corresponding black holes solutions and their thermodynamic properties are interesting areas of studies from the point of view of such generalized scalar coupling. It may be recalled that the thermodynamic properties of various black holes have been extensively investigated in different context 9, 10, 11, 12, 13, 14, 15, 16, 17, 18, 19, 20, 21, 22, 23, 24, 25, 26, 27, 28, 29, 30, 31, 32, 33, 34, 35, 36, 37, 38, 39, 40, 41, 42, 43, 44, 45, 46. In this work we shall study the thermodynamic properties of the asymptotically flat dilaton-axion black holes as obtained from the above action [8] . After deriving the general expressions for the Hawking temperature and entropy for arbitrary value of $a$ and $b$, we shall consider two different cases : $|b|=|a|$, which implies that the dilaton coupling parameter $a$ and the axion coupling parameter $b$ with electromagnetic field are equal, and $|b| \neq|a|$ with $b<<1$ implying that the axion coupling is much weaker. Area-entropy law as well as first law of black hole mechanics are verified

\footnotetext{
* E-mail: tanwi.ghosh@yahoo.co.in

$\dagger$ E-mail: tpssg@iacs.res.in
} 
for these class of black holes. We shall further show that these two cases yield distinct results in terms of thermodynamic properties of black holes, indicating the different roles of the scalar and pseudo scalars in determining the thermodynamic properties. Our result brings out that with increase in axion coupling, the black hole may undergo second order phase transition.

\section{Asymptotically Flat Dilaton-Axion Black hole}

Considering above mentioned Einstein-Maxwell-Kalb-Ramond action for arbitrary coupling parameters a and b, static spherically symmetric black hole solution is given by [8],

$$
d s^{2}=-\frac{\left(r-r_{+}\right)\left(r-r_{-}\right)}{\left(r-r_{0}\right)^{2-2 n}\left(r+r_{0}\right)^{2 n}} d t_{s}^{2}+\frac{\left(r-r_{0}\right)^{2-2 n}\left(r+r_{0}\right)^{2 n}}{\left(r-r_{+}\right)\left(r-r_{-}\right)} d r^{2}+\frac{\left(r+r_{0}\right)^{2 n}}{\left(r-r_{0}\right)^{(2 n-2)}}\left[d \theta^{2}+\sin ^{2} \theta d \phi^{2}\right]
$$

where electromagnetic field components and black hole horizons are

$$
\begin{aligned}
& F_{t r}=\frac{\left(q_{e}-q_{m} b \zeta\right) e^{a \varphi}}{\left(r-r_{0}\right)^{2(1-n)}\left(r+r_{0}\right)^{2 n}} d t d r \text { and } F_{\theta \phi}=q_{m} \sin \theta d \theta d \phi \\
& r_{ \pm}=m_{0} \pm \sqrt{m_{0}^{2}+r_{0}^{2}-\frac{1}{8}\left(\frac{K_{1}}{n}+\frac{K_{2}}{1-n}\right)} ; r_{0}=\frac{1}{16 m_{o}}\left(\frac{K_{1}}{n}-\frac{K_{2}}{1-n}\right) \\
& m_{0}=m-(2 n-1) r_{0} ; K_{1}=4 n\left[4 r_{0}^{2}+2 k r_{0}\left(r_{+}+r_{-}\right)+k^{2} r_{+} r_{-}\right] ; K_{2}=4(1-n) r_{+} r_{-} ; 0<n<1 \text { and } \\
& m=\frac{1}{16 r_{0}}\left(\frac{K_{1}}{n}-\frac{K_{2}}{1-n}\right)+(2 n-1) r_{0}, \text { where } m \text { is the mass of the black hole and } \mathrm{k}=1 \text { for asymptotically flat }
\end{aligned}
$$
case.

For these class of black hole, the surface gravity $\kappa$ can be easily obtained as,

$$
\kappa=\frac{\left(r_{+}-r_{-}\right)}{\left(r_{0}+r_{+}\right)^{2 n}\left(r_{+}-r_{0}\right)^{2-2 n}}
$$

This gives the the Hawking temperature $\mathrm{T}$ of the black hole as,

$$
T=\frac{\kappa}{2 \pi}=\frac{\left(r_{+}-r_{-}\right)}{4 \pi\left(r_{0}+r_{+}\right)^{2 n}\left(r_{+}-r_{0}\right)^{2-2 n}}
$$

In order to determine the entropy, the expression of area $A$ can be derived from,

$$
A=\int \sqrt{g_{\theta \theta} g_{\phi \phi}} d \theta d \phi
$$

For the above metric this yields,

$$
=4 \pi \frac{\left(r_{+}+r_{0}\right)^{2 n}}{\left(r_{+}-r_{0}\right)^{2 n-2}}
$$

To study further thermodynamic behaviour as well as phase transitions for such dilaton-axion black hole, we now consider the following special cases in respect to dilaton and axion coupling parameters $a$ and $b$.

Case - I : $a=b$

This case corresponds to the solution when the dilaton and axion couples with equal strength with the Maxwell field. For asymptotically flat case we begin with this condition i.e. $|a|=|b|$.In this case black hole metric solution takes the form [8];

$$
d s^{2}=-\left(1-\frac{2 m_{0}}{r}\right)\left(1-\frac{2 r_{0}}{r}\right)^{\frac{1-a^{2}}{1+a^{2}}} d t^{2}+\left(1-\frac{2 m_{0}}{r}\right)^{-1}\left(1-\frac{2 r_{0}}{r}\right)^{\frac{a^{2}-1}{a^{2}+1}} d r^{2}+r^{2}\left(1-\frac{2 r_{0}}{r}\right)^{\frac{2 a^{2}}{1+a^{2}}} d \Omega^{2}
$$

where parameters $m_{0}, r_{0}$, the black hole mass $\mathrm{m}$ total charge Q,electric charge $Q_{e}$ and magnetic charge $Q_{m}$ are related as: $r_{0}=\frac{\left(1+a^{2}\right) Q^{2} e^{-a \varphi_{0}}}{4 m_{0}}, m_{0}=m-\frac{\left(1-a^{2}\right)}{\left(1+a^{2}\right)} r_{0}, Q^{2}=Q_{e}^{2}+Q_{m}^{2}$ with $\varphi_{0}$ as the asymptotic value of the scalar field. These solutions represent black holes with it's horizon located at $r=r_{+}=2 m_{0}$. 
Such solution clearly has a curvature singularity at $r=2 r_{0}$.

The action for this spherically symmetric dilaton-axion black hole has the well-known form [24]

$$
I_{a c}=\frac{\beta}{2}\left(m-Q_{e} \Phi\right)
$$

where $Q_{e}$ is the electric charge with the corresponding potential $\Phi=\frac{Q_{e} e^{-a \varphi_{0}}}{r_{+}}$.Substituting for $r_{+}$and $\mathrm{m}$ the action becomes,

$$
I_{a c}=\frac{\beta}{2}\left(m_{0}-\frac{Q_{e}^{2} e^{-a \varphi_{0}}}{2 m_{0}}+\frac{\left(1-a^{2}\right) r_{0}}{\left(1+a^{2}\right)}\right)
$$

The thermodynamic potential $\mathrm{W}$ for the corresponding grand canonical ensemble is given as, $W=E-$ $T S-Q_{e} \Phi$, where $\mathrm{S}$ and $\mathrm{E}$ are the entropy and energy of the black hole. From the expressions of the action we can find out thermodynamic quantities as follows [24, 30]:

$$
W=\frac{I_{a c}}{\beta}=\frac{1}{2}\left(m_{0}-\frac{Q_{e}^{2} e^{-a \varphi_{0}}}{2 m_{0}}+\frac{\left(1-a^{2}\right)}{\left(1+a^{2}\right)} r_{0}\right)
$$

The potential $\Phi$ has the well known form

$$
\Phi=\left(\frac{\partial W}{\partial Q_{e}}\right)_{\beta}=\frac{Q_{e} e^{-a \varphi_{0}}}{2 m_{0}}=\frac{Q_{e} e^{-a \varphi_{0}}}{r_{+}}
$$

Using equation (5) this becomes,

$$
\Phi=\frac{Q_{e} e^{-a \varphi_{0}}}{2 m_{0}}=\frac{Q_{e} e^{-a \varphi_{0}}}{r_{+}}
$$

The corresponding free energy

$$
F=E-T S=W+Q_{e} \Phi=\frac{1}{2}\left(m_{0}+\frac{Q_{e}^{2} e^{-a \varphi_{0}}}{2 m_{0}}+\frac{\left(1-a^{2}\right)}{\left(1+a^{2}\right)} r_{0}\right)
$$

and the surface gravity

$$
\kappa=\frac{2 m_{0}}{r_{+}^{2}}\left(1-\frac{2 r_{0}}{r_{+}}\right)^{\frac{\left(1-a^{2}\right)}{\left(1+a^{2}\right)}}
$$

The Hawking temperature of the black hole is now easily determined as,

$$
T=\frac{\kappa}{2 \pi}=\frac{1}{4 \pi} \frac{2 m_{0}}{r_{+}^{2}}\left(1-\frac{2 r_{0}}{r_{+}}\right)^{\frac{\left(1-a^{2}\right)}{\left(1+a^{2}\right)}}=\frac{1}{4 \pi} \frac{1}{r_{+}}\left(1-\frac{2 r_{0}}{r_{+}}\right)^{\frac{\left(1-a^{2}\right)}{\left(1+a^{2}\right)}}
$$

Now using [37, 45, 46]

$$
S=\beta^{2}\left(\frac{\partial F}{\partial \beta}\right)_{Q_{e}}
$$

one obtains

$$
S=\pi r_{+}^{2}\left(1-\frac{2 r_{0}}{r_{+}}\right)^{\frac{2 a^{2}}{\left(1+a^{2}\right)}}
$$

To verify the Bekenstein-Hawking [27] area-entropy law, the area A of the black hole can now be derived as,

$$
A=\int \sqrt{g_{\theta \theta} g_{\phi \phi}} d \theta d \phi=4 \pi r_{+}^{2}\left(1-\frac{2 r_{0}}{r_{+}}\right)^{\frac{2 a^{2}}{\left(1+a^{2}\right)}}
$$


Comparing with the expression of entropy above, we find that entropy S and area A are related by

$$
S=\frac{A}{4}
$$

This confirms the Bekenstein-Hawking area-entropy law for these new class of scalar coupled black holes. Substituting the expressions of $r_{0}$ and $r_{+}$,one can further write the entropy as,

$$
S=\pi r_{+}^{2}\left[1-\frac{\left(1+a^{2}\right) Q_{e}^{2} e^{-a \varphi_{0}}}{4 m_{0}^{2}}\right]^{\frac{2 a^{2}}{\left(1+a^{2}\right)}}
$$

From the above expressions of temperature,entropy and potential we immediately obtain,

$$
T d S+\Phi d Q_{e}=d m
$$

where we have substituted $\frac{Q_{e}^{2} e^{-a \varphi_{0}}}{2 m_{0}}=\frac{2 r_{0}}{\left(1+a^{2}\right)}$. This reconfirms the first law of black hole thermodynamics in this context.

For static spherically symmetric black hole,using $E=2 T S+Q \Phi$,action $I_{a c}$ can be written as,

$$
I_{a c}=\frac{\beta}{2}\left(m_{0}+\frac{\left(1-a^{2}\right) r_{0}}{\left(1+a^{2}\right)}-\frac{Q_{e}^{2} e^{-a \varphi_{0}}}{2 m_{0}}\right)=\frac{\beta}{2} m_{0}\left(1-\frac{r_{0}}{m_{0}}\right)=\pi r_{+}^{2}\left(1-\frac{2 r_{0}}{r_{+}}\right)^{\frac{2 a^{2}}{\left(1+a^{2}\right)}}
$$

which is same as the expression of the entropy. This leads to $I_{a c}=S$. Moreover thermodynamically black hole contribution to the energy is defined as,

$$
E=\left(\frac{\partial(\beta F)}{\partial \beta}\right)_{Q_{e}}=m
$$

We now look into the properties of the specific heat of such black holes. Using the well-known expression for the specific heat,

$$
C_{Q}=T\left(\frac{\partial S}{\partial T}\right)_{Q}
$$

the specific heat in this case becomes,

$$
\begin{aligned}
C_{Q} & =T\left[2 \pi r_{+}\left(1-\frac{2 r_{0}}{r_{+}}\right)^{\frac{2 a^{2}}{\left(1+a^{2}\right)}}\right. \\
& \left.+\frac{4 a^{2} \pi 2 r_{0}}{\left(a^{2}+1\right)}\left(1-\frac{2 r_{0}}{r_{+}}\right)^{\frac{\left(a^{2}-1\right)}{\left(a^{2}+1\right)}}\right]\left[\frac{-1}{4 \pi r_{+}^{2}}\left(1-\frac{2 r_{0}}{r_{+}}\right)^{\frac{\left(-a^{2}+1\right)}{\left(a^{2}+1\right)}}+\frac{1}{4 \pi r_{+}} \frac{2\left(-a^{2}+1\right)}{\left(a^{2}+1\right)} \frac{2 r_{0}}{r_{+}^{2}}\left(1-\frac{2 r_{0}}{r_{+}}\right)^{\frac{-2 a^{2}}{\left(1+a^{2}\right)}}\right]^{-1} \\
& =-2 \pi r_{+}^{2}\left(1-\frac{2 r_{0}}{r_{+}}\right)^{\frac{2 a^{2}}{\left(1+a^{2}\right)}}\left[1-\frac{\left(1-a^{2}\right) Q_{e}^{2} e^{-a \varphi_{0}}}{4 m_{0}^{2}}\right]\left[1-\frac{\left(3-a^{2}\right) Q_{e}^{2} e^{-a \varphi_{0}}}{4 m_{0}^{2}}\right]^{-1}
\end{aligned}
$$

We now analyse the above expression of specific heat for different regime in the parameter space. From the expression of entropy it follows that the entropy will be non-negative if $\frac{\left(1+a^{2}\right) Q_{e}^{2} e^{-a \varphi_{0}}}{4 m_{0}^{2}}<1$. This in turn implies that $\frac{\left(1-a^{2}\right) Q_{e}^{2} e^{-a \varphi_{0}}}{4 m_{0}^{2}}<1$. Therefore for $\frac{\left(3-a^{2}\right) Q_{e}^{2} e^{-a \varphi_{0}}}{4 m_{0}^{2}}<1$, specific heat is negative. Such black holes therefore can not be stable locally.

However for $\frac{\left(3-a^{2}\right) Q_{e}^{2} e^{-a \varphi_{0}}}{4 m_{0}^{2}}>1$ along with $\frac{\left(a^{2}+1\right) Q_{e}^{2} e^{-a \varphi_{0}}}{4 m_{0}^{2}}<1$, one finds that $a^{2}<1$ and in such a scenario the specific heat clearly becomes positive and the black hole is stable.

Moreover for

$$
\frac{\left(3-a^{2}\right) Q_{e}^{2} e^{-a \varphi_{0}}}{4 m_{0}^{2}}=1
$$

the specific heat blows up while the temperature and the entropy continue to be finite. This signals a second order phase transition for such black holes. The phase transition therefore occurs when the charge 
to mass ratio of the black hole is related to the coupling parameter $a$ and the scalar vacuum expectation

It is interesting to observe that when $\frac{\left(a^{2}+1\right) Q_{e}^{2} e^{-a \varphi_{0}}}{4 m_{0}^{2}}=1$, the temperature,entropy as well as the specific heat of the black hole becomes zero.

It may further be noted that for $r_{0}=0$, the expression of specific heat reduces to that for a Schwarzschild black hole as is expected.

\section{Case - II : $a \neq b$}

Extending our discussion for the asymptotically flat case, we now consider a different region in the parameter space of $a$ and $b$ for which $|a| \neq|b|$ with $a=1$ and $b<<1$. This implies that the axion coupling charge to the Maxwell field is much smaller than the dilaton coupling charge. The corresponding metric is given as, [8],

$$
d s^{2}=-\frac{\left(r-r_{+}\right)\left(r-r_{-}\right)}{r^{2}-r_{0}^{2}} d t^{2}+\frac{r^{2}-r_{0}^{2}}{\left(r-r_{+}\right)\left(r-r_{-}\right)} d r^{2}+\left(r^{2}-r_{0}^{2}\right) d \Omega^{2}
$$

where $r_{0}=\frac{\left(Q_{e}^{2}-Q_{m}^{2}\right) e^{-\phi_{0}}}{2 m}$ and event horizons are located at $r_{ \pm}=m \pm \sqrt{m^{2}+r_{0}^{2}-\left(Q_{e}^{2}+Q_{m}^{2}\right) e^{-\phi_{0}}}$. In this case the action can be written as,

$$
I_{a c}=\frac{\beta}{2}\left(m-Q_{e} \Phi\right)
$$

where potential $\Phi=\frac{Q_{e} e^{-\phi_{0}}}{\left(r_{+}+r_{0}\right)}, r_{0}=\frac{Q_{e}^{2} e^{-\phi_{0}}}{2 m}$.

The corresponding grand canonical potential W can be obtained from the action as,

$$
W=\frac{I_{a c}}{\beta}=\frac{1}{2}\left(m-\frac{Q_{e}^{2} e^{-\phi_{0}}}{2 m}\right)
$$

The free energy function $\mathrm{F}$ turns out to be,

$$
F=E-T S=W+Q_{e} \Phi=\frac{1}{2}\left(m+\frac{Q_{e}^{2} e^{-\phi_{0}}}{2 m}\right)
$$

From these the expression of entropy for this black hole can be obtained as

$$
S=\beta^{2}\left(\frac{\partial F}{\partial \beta}\right)_{Q_{e}}=\pi\left(r_{+}^{2}-r_{0}^{2}\right)
$$

Calculation of horizon area $A$ in this case yields,

$$
A=\int \sqrt{g_{\theta \theta} g_{\varphi \varphi}} d \theta d \varphi=4 \pi\left(r_{+}^{2}-r_{0}^{2}\right)
$$

Thus once again equations(24)and (25) confirms the Bekenstein-Hawking [27] area-entropy law for black hole mechanics. Substituting the expressions of $r_{+}$and $r_{0}$, the expression for entropy reduces to,

$$
S=\pi 4 m^{2}\left(1-\frac{Q_{e}^{2} e^{-\varphi_{0}}}{2 m^{2}}\right)
$$

Calculating the surface gravity, $\kappa$ one finds,

$$
\kappa=\frac{\left(r_{+}-r_{-}\right)}{\left(r_{+}^{2}-r_{0}^{2}\right)}
$$

From this the temperature of the black hole is found to be,

$$
T=\frac{\kappa}{2 \pi}=\frac{1}{4 \pi} \frac{\left(r_{+}-r_{-}\right)}{\left(r_{+}^{2}-r_{0}^{2}\right)}
$$


Combining all these together one arrives at the first law of black hole mechanics,

$$
T d S+\Phi d Q_{e}=d m
$$

Similar to the Case I, energy of the black hole is $\mathrm{E}=\mathrm{m}$.

Substituting the expression of $\beta, \mathrm{m}$ and $\Phi Q_{e}$, once again the action can be written in terms of entropy $\mathrm{S}$ as

$$
I_{a c}=\pi\left(r_{+}^{2}-r_{0}^{2}\right)=S
$$

Finally using the expression for specific heat as mentioned in CaseI, we find

$$
C_{Q}=\frac{-1}{8 \pi m}(8 \pi m)\left(16 \pi m^{2}\right)=-4 \pi\left(r_{+}+r_{0}\right)^{2}
$$

The specific heat $C_{J, Q}$ for such black hole is therefore always negative. So this kind of black hole is never stable locally. Once again it is easy to see that for $r_{0}=0$ the expression for $C_{J, Q}$ reduces to that for a Schwarzschild black hole.

It is important to observe that in Case-II, we have considered the axion coupling parameter $b$ to be very weak and much smaller than the dilaton coupling parameter $a$. Unlike Case- I, the black hole is never stable and no phase transition occurs in this case, at least classically. This establishes an interesting role of the pseudo scalar axion in determining the thermodynamic properties of black holes. Our result reveals that whenever the pseudo sclar coupling becomes as strong as the scalar coupling, the black hole can indeed go through second order phase transition and can therefore achieve local stability.

\section{Conclusion}

Various thermodynamic properties for a dilaton-axion coupled black hole solutions are determined for a more general class of their electromagnetic coupling parameters $a$ and $b$. It is found that for different regions in the coupling parameter space, the thermodynamic properties are distinct. The BekensteinHawking area-entropy law as well as the first law of black hole thermodynamics have been verified for these class of scalar coupled black holes. This once again ensures the generality of the area-entropy law.

The issue of stability of such black holes have been examined. Analysing the properties of the specific heat it is shown that while for certain region in the coupling parameter space the black hole can be both stable and unstable depending on the value of it's mass to charge ratio and the scalar vacuum expectation value, for a different choice of couplings they are unstable irrespective of the value of it's charge or mass. Furthermore it has been shown that a certain relation between the mass-charge ratio and the scalar vacuum expectation value signals the occurrence of a second order phase transition. The constraint on the scalar coupling parameter $a$ in such situation is determined. It is shown that in the region where axion coupling parameter is much smaller than the dilaton coupling parameter one find the specific heat of the black hole to be always negative. Such unstable black hole can become stable through a phase transition when the axion coupling parameter becomes larger and comparable to the dilaton coupling parameter . This clearly brings out the effects of scalar and pseudo scalar couplings on the thermodynamic properties of black holes. Our result reveals that even at the classical level, an appropriate choice of the axion coupling can result into a second order phase transition.

[1] D.Garfinkle,G.T.Horowitz and A.Strominger,Phys.Rev.D43,3140,1991.

[2] A.D.Shapere,S.Trivedi and F.Wilczek,Mod.Phys.Lett.A6,2677,1991.

[3] A.Sen,Nucl.Phys.B404,109,1993.

[4] A.Sen,Phys.Rev.Lett69,1006,1992.

[5] T.Ortin,Phys.Rev.D47,3136,1993.

[6] R.Kallosh and T.Ortin,Phys.Rev.D48,742,1993.

[7] G.T.Horrowitz and A.Strominger,Nucl.Phys.B360,197,1991.

[8] S.Sur, S.Das and S.Sengupta,JHEP 0510:064,2005.

[9] P.C.W.Davies,Proc.Roy.Soc.LondA353,499,1977.

[10] G.Ruppeiner,Phys.Rev.D75,024037,2007. 
[11] Jan E.Aman,Narit Pidokrajt,Phys.Rev.D73,024017,2006.

[12] Yun Soo Myung,Phys.Rev.D77,104007,2008,Yun Soo Myung,Yong-wan Kim,Young-Jai Park arXiv:0708.3145.

[13] Jose L Alvarez,F.Quevedo and A.Sanchez,Phys.Rev.D77,084004,2008.

[14] T.Ghosh,JCAP 0411:003,2004.

[15] Don N Page,New J Phys.7,203,2005,Class.Quant.Grav6,1909,1989.

[16] S.Das,Pramana63,797,2004.

[17] V. Berezin,Nucl.Phys.B661,409,2003.

[18] E. Radu,Mod.Phys.Lett A17,2277,2002.

[19] T. Pilling,Phys.Lett B660,402,2008.

[20] Shuang-Quing Wu,Phys.Lett B608,251,2005.

[21] B.B.Wang and C.G.Huang,Class.Quant.Grav19,2491,2002.

[22] Ju-Hua Chen,Ji-Liang Jing and Yong-Jiu Wang,Chin.Phys.10,467,2001.

[23] Ji-liang Jing,Chin.Phys.Lett14,81,1997.

[24] Ji-liang Jing,Nucl.Phys.B476,548,1996.

[25] James W. York,Jr,Phys.Rev.D33,2092,1986.

[26] Sergey.N Solodukhin,Phys.Rev.D54,3900,1996.

[27] J.D.Bekenstein,Phys.Rev.D12,3077,1975.

[28] Chamblin,Emparan,Johnson and Myers,Phys.Rev.D60,104026,1999, Phys.Rev.D60,064018,1999.

[29] Hawking and Page,Commun.Math.Phys87,577,1983.

[30] Caldarelli,Cognola and Klemm,Class.Quant.Grav17,399,2000.

[31] P.Mitra,Phys.Lett.B441,89,1998,Phys.Lett.B459,119,1999.

[32] Peca and Lemos,Phys.Rev.D59,124007,1999.

[33] Louko and Winters-Hilt,Phys.Rev.D54,2647,1999.

[34] Hawking,"Stability in Ads and Phase Transitions",talk given at Strings 99,Potsdam (Germany),july 19-24,1999.

[35] Berman and Parikh,Phys.Lett.B463,168,1999.

[36] M.Cvetic and S.S.Gubsar,JHEP07,010,1999.

[37] G't Hooft,Nucl.Phys.B256,727,1985.

[38] J.M.Bardeen,B.Carter and S.W Hawking,Commun.Math.Phys31,61,1973.

[39] L.Smarr,Phys.Rev.Lett30,71,1973.

[40] H.W.Braden,J.D.Brown,B.F. Whiting and J.W.Yory,Jr,Phys.Rev.D42,3376,1990.

[41] B.F.Whiting and J.W.York,Jr,Phys.Rev.Lett61,1336,1988.

[42] A.Sheykhi,N Riazi and M.H.Mahzoon,Phys.Rev.D74,044025,2006.

[43] Tower Wang,Nucl.Phys.B756,86,2006.

[44] S.S.Gubser, Nucl.Phys.B551,667,1999.

[45] T.Ghosh, Mod.Phys.Lett A 22, 2865, 2007

[46] T.Ghosh, S.SenGupta,Phys.Rev.D78,024045,2008. 